\title{
Excitability of Spinal Neurons during Relaxation Imagery for 2 Minutes
}

Toshiaki Suzuki ${ }^{1,2 *}$, Yoshibumi Bunno ${ }^{2}$, Chieko Onigata², Makiko Tani ${ }^{1,2}$, Hirohisa Yoneda ${ }^{1,2}$, Takaki Yoshida ${ }^{2}$, Yoshitsugu Tanino ${ }^{1,2}$ and Sayuri Uragami²

${ }^{1}$ Graduate School of Health Sciences, Graduate School of Kansai University of Health Sciences, Japan

${ }^{2}$ Clinical Physical Therapy Laboratory, Faculty of Health Sciences, Kansai University of Health Sciences, Japan

\begin{abstract}
We investigated spinal neuron function during relaxation imagery by analyzing F-waves generated from the left thenar muscles in response to stimulation of the left median nerve at the wrist in 10 healthy subjects. F-waves were recorded in a resting position and during, immediately after, and at 5, 10, and $15 \mathrm{~min}$ after a 2-min relaxation imagery period. Persistence, F/M amplitude ratio, and latency were analyzed from the recorded F-waves.
\end{abstract}

The F/M amplitude ratio was lower in the relaxation imagery condition than in the resting condition, whereas it either recovered or continued to decrease after relaxation imagery. There were no significant differences in persistence and latency before, during, and after relaxation imagery.

These results suggested that 2-min relaxation imagery can decrease spinal neural function in healthy subjects and may be useful as a new type of physical therapy for patients suffering from impaired motor control.

Keywords: F-waves; Spinal neurons; F/M amplitude ratio; Relaxation imagery; Physical therapy

\section{Introduction}

Physical therapy using relaxation imagery, which involves active relaxation of the brain, is often used to decrease the abnormally increased muscle tone in patients with spasticity and rigidity. We have experienced cases where relaxation imagery was effective in patients with poor muscle control due to neurological diseases.

In our previous study, we investigated spinal neuron excitability in healthy subjects by analyzing F-waves before, during, and after a 1-min period of relaxation imagery [1]. There were no significant differences in F-waves obtained at rest and during and after relaxation imagery. The reason for this was thought to be the short period of relaxation imagery (1 min).

Therefore, the current study investigated spinal neuron excitability in healthy subjects by analyzing F-waves generated before, during, and after a 2-min period of relaxation imagery, which was expected to have greater effects considering its longer duration.

\section{Materials and Methodology}

\section{Subjects}

Ten healthy subjects ( 7 males, 3 females; mean age, $34.8 \pm 10.2$ years) participated in the study. Written informed consent was obtained from all subjects. This study was approved by the Research Ethics Committee at Kansai University of Health Sciences. The experiments were conducted in accordance with the Declaration of Helsinki. The authors of this study report no conflicts of interest.

\section{F-waves during relaxation imagery}

All subjects were positioned in a comfortable supine position with external rotation of both shoulder joints. The skin was prepared with an abrasive gel to maintain the impedance below $5 \mathrm{~K} \Omega$. A Viking Quest electromyography machine (Natus Medical Inc., CA, USA) was used to record F-waves. We measured F-waves generated from the left thenar muscles using a pair of round disks attached to the skin with collodion and placed over the belly and on the bone of the metacarpal-phalangeal joint of the thumb. Measurements were obtained after stimulation of the left median nerve at the wrist at rest and under the relaxation imagery condition. The stimulating electrodes comprised a cathode placed over the left median nerve $3 \mathrm{~cm}$ proximal to the palmar crease of the wrist joint and an anode placed a further $2 \mathrm{~cm}$ proximally. The maximal stimulus was determined by delivering $0.2-\mathrm{ms}^{2}$ wave pulses of increasing intensity to elicit the largest compound muscle action potential. Supramaximal shocks (adjusted up to $20 \%$ higher than the maximal stimulus) were delivered at $0.5 \mathrm{~Hz}$ for $\mathrm{F}$-wave acquisition. The bandwidth filter was $2-3 \mathrm{kHz}$.

Under the resting condition, we measured F-waves during relaxation. Under the relaxation imagery condition, F-waves were recorded in a resting position and during, immediately after, and at 5 , 10 , and $15 \mathrm{~min}$ after a 2 -min relaxation imagery period. Our method of relaxation imagery was to relax the left thenar muscles because we concluded, in our previous study, that the best method may be to focus on the body part that needs to be relaxed.

\section{Data analysis}

F-waves generated during 30 trials were analyzed for persistence, $\mathrm{F} / \mathrm{M}$ amplitude ratio, and latency. Persistence was defined as the number of measurable F-wave responses divided by 30 trials of supramaximal stimulation. The $\mathrm{F} / \mathrm{M}$ amplitude ratio was defined as the mean amplitude of all responses divided by the M-wave amplitude. Latency was defined as the mean latency from the time of stimulation to the onset of a measurable F-wave.

Dunnett's test was used to compare results between the resting and relaxation imagery conditions.

\section{Results}

The F/M amplitude ratio was lower in the relaxation imagery

*Corresponding author: Toshiaki Suzuki, Graduate School of Health Sciences, Graduate School of Kansai University of Health Sciences, 2-11-1 Wakaba, Kumatori, Sennan, Osaka 590-0482, Japan, Tel: +81-72-453-8374; Fax: +81-72-453-8798; E-mail: suzuki@kansai.ac.jp

Received March 27, 2014; Accepted Aprile 25, 2014; Published May 30, 2014

Citation: Suzuki T, Bunno Y, Onigata C, Tani M, Yoneda H, et al. (2014) Excitability of Spinal Neurons during Relaxation Imagery for 2 Minutes. Int J Neurorehabilitation 1: 105. doi:10.4172/2376-0281.1000105

Copyright: @ 2014 Suzuki T, et al. This is an open-access article distributed under the terms of the Creative Commons Attribution License, which permits unrestricted use, distribution, and reproduction in any medium, provided the original author and source are credited. 


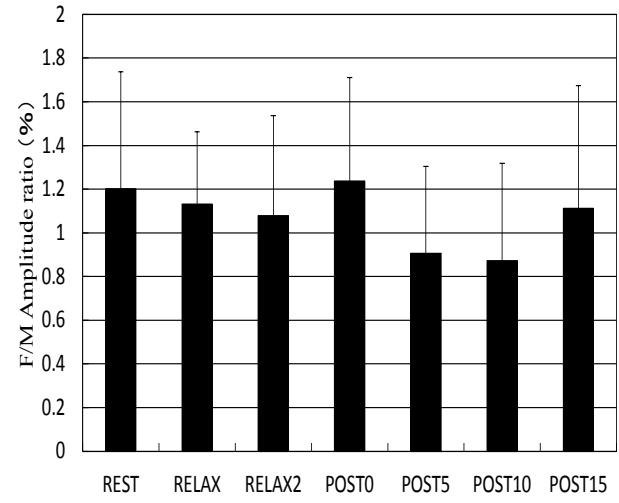

rest: resting position, relax: under relaxation imagery from 0 to 1 min, relax2: under relaxation imagery from 1 to 2 min, post 0 : immediately after imagery, post5: 5 min after imagery, post10: 10 min after imagery, post15: 15min after imagery

Figure 1: The F/M amplitude ratio in the resting position and during, immediately after, and at 5, 10, and $15 \mathrm{~min}$ after a 2-min period of relaxation imagery.

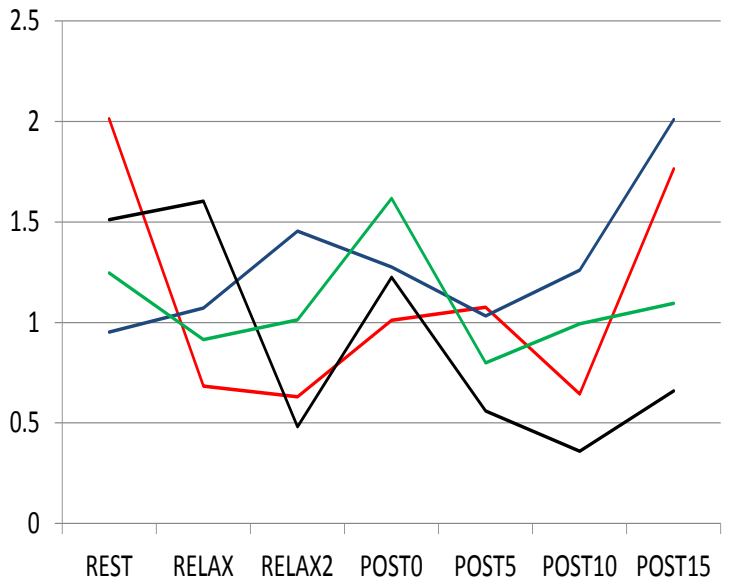

rest: resting position, relax: under relaxation imagery from 0 to 1 min, relax2: under relaxation imagery from 1 to $2 \mathrm{~min}$, post0: immediately after imagery, post5: 5 min after imagery, post10: 10 min after imagery, post15: $15 \mathrm{~min}$ after imagery

Figure 2: Typical patterns of changes in the $\mathrm{F} / \mathrm{M}$ amplitude ratio under the resting condition, under relaxation imagery for $2 \mathrm{~min}$, and after the imagery.

condition than in the resting condition (Figure 1). Figure 2 shows 4 typical patterns of changes in the $\mathrm{F} / \mathrm{M}$ amplitude ratio during relaxation imagery. The red line shows a decreasing pattern during relaxation imagery (4/10 subjects). The green line shows a pattern of decrease from 0 to $1 \mathrm{~min}$ and increase from 1 to $2 \mathrm{~min}$ (1/10 subjects). The black line shows a pattern of increase from 0 to $1 \mathrm{~min}$ and decrease from 1 to 2 min (4/10 subject). The blue line shows a pattern of gradual increase over the 2 -min period (1/10 subjects). Figure 2 shows that almost of subjects tended to show a decrease in $\mathrm{F} / \mathrm{M}$ amplitude ratio during the 2-min relaxation imagery period.

Immediately after relaxation imagery, the $\mathrm{F} / \mathrm{M}$ amplitude ratio tended to increase, following which it tended to decrease (Figure 1). However, individual values for $\mathrm{F} / \mathrm{M}$ amplitude ratios after relaxation imagery were varied (Figure 2).
There were no significant differences in persistence and latency before, during, and after relaxation imagery (Figures 3,4).

\section{Discussion}

Techniques such as continued muscle stretching [2,3] and neuromuscular electrical stimulation [4] are usually employed to decrease abnormal muscle tone in patients with spasticity and rigidity.

Relaxation imagery was previously found to be effective in improving psychological well-being measures and stress scale scores $[5,6]$. Few studies have used relaxation imagery as a method for decreasing abnormal muscle tone. In our previous study, we investigated spinal neuron excitability in healthy subjects by measuring F-waves at rest and during a 1-min period of relaxation imagery [1] and found that relaxation imagery for $1 \mathrm{~min}$ did not decrease spinal neuron excitability [1].

In contrast, this study demonstrated decreased spinal neural

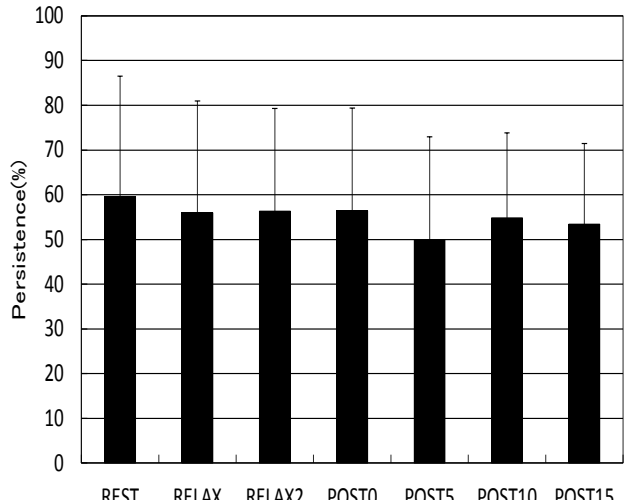

rest: resting position, relax: under relaxation imagery from 0 to $1 \mathrm{~min}$, relax2: under relaxation imagery from 1 to 2 min, post 0 : immediately after imagery, post5: 5 min after imagery, post10: 10 min after imagery, post15: $15 \mathrm{~min}$ after imagery

Figure 3: Persistence in the resting position and during, immediately after, and at 5,10 , and $15 \mathrm{~min}$ after a 2 -min period of relaxation imagery.

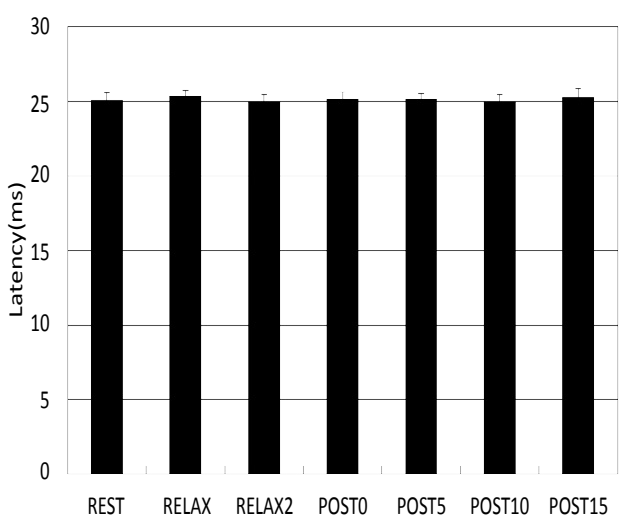

rest: resting position, relax: under relaxation imagery from 0 to 1 min, relax2: under relaxation imagery from 1 to 2 min, post 0 : immediately after imagery, post5: 5 min after imagery, post10: 10 min after imagery, post15: 15min after imagery

Figure 4: Latency in the resting position and during, immediately after, and at 5,10 , and $15 \mathrm{~min}$ after a 2-min period of relaxation imagery. 
Citation: Suzuki T, Bunno Y, Onigata C, Tani M, Yoneda H, et al. (2014) Excitability of Spinal Neurons during Relaxation Imagery for 2 Minutes. Int J Neurorehabilitation 1: 105. doi:10.4172/2376-0281.1000105

function during a 2-min period of relaxation imagery, indicating that 2 min of relaxation imagery is more effective than 1 min for suppressing excitability of the central nervous system (CNS). Furthermore, extending the period of relaxation imagery to $2 \mathrm{~min}$ can be effective in decreasing abnormal muscle tone.

Immediately after relaxation imagery for $2 \mathrm{~min}$, the $\mathrm{F} / \mathrm{M}$ amplitude ratio tended to increase and then decrease. This indicated that the recovery of spinal neural function was facilitated immediately after relaxation imagery, following which it decreased again. However, in our previous report using a motor imagery task in which the subjects were asked to imagine $50 \%$ maximum voluntary contraction (MVC) during isometric contraction, the $\mathrm{F} / \mathrm{M}$ amplitude ratio did not change after motor imagery [7]. In this study, relaxation imagery for 2 min could evoke continued inhibition of spinal neural function after relaxation imagery.

In the future, we would like to study the effect of relaxation imagery using F-wave to reduce the muscle tone for spasticity in patients with cerebrovascular diseases.

\section{Conclusions}

We concluded that relaxation imagery for 2 min has the potential to decrease spinal neural function in healthy subjects. This study demonstrated decreased spinal neural function during a 2-min period of relaxation imagery, indicating that $2 \mathrm{~min}$ of relaxation imagery is more effective than $1 \mathrm{~min}$ for suppressing excitability of the central nervous system (CNS) in our previous report. We believe that relaxation imagery for decreasing muscle tone may be useful as a new type of physical therapy for patients with spasticity and rigidity.

\section{Conflicts of Interest} study.

The authors declare that there were no conflicts of interest in this

\section{References}

1. Suzuki T, Bunno Y, Onigata C, Tani M, Uragami S (2014) Excitability of spinal neurons during a short period of relaxation imagery. The Open General \& Internal Medicine Journal 6:1-5

2. Suzuki T, Saitoh E, Tani M, Nabeta R, Daikuya S, et al. (2003) Effect of continued stretching of the affected arm in patients with cerebrovascular disease by examining $\mathrm{H}$-reflex characteristics. Electromyogr Clin Neurophysiol 43: $51-56$.

3. Suzuki T, Saitoh E, Daikuya S, Hirose H, Tani M, et al. (2003) Characteristics of F-wave in different stretched position of the affected arm in patients with cerebrovascular diseases. Electromyogr Clin Neurophysiol 43: 97-101.

4. Stowe AM, Hughes-Zahner L, Barnes VK, Herbelin LL, Schindler-Ivens SM, et al. (2013) A pilot study to measure upper extremity H-reflexes following neuromuscular electric stimulation therapy after stroke. Neurosci Lett 535: 1-6.

5. Nelson EA, Dowsey MM, Knowles SR, Castle DJ, Salzberg MR, et al. (2013) Systematic review of the efficacy of pre-surgical mind-body based therapies on post-operative outcome measures. Complement Ther Med 21: 697-711.

6. Dawson MA, Hamson-Utley JJ, Hansen R, Olpin M (2014) Examining the Effectiveness of Psychological Strategies on Physiologic Markers: EvidenceBased Suggestions for Holistic Care of the Athlete. J Athl Train .

7. Bunno $Y$, Yurugi Y, Onigata C, Suzuki T, Iwatsuki H. Influence of Motor Imagery of Isometric Opponens Pollicis Activity on the Excitability of Spinal Motor Neurons: Comparison using Different Muscle Contraction Strengths. Journal of Physical Therapy Science (in press). 